\title{
COMPOSITION OPERATORS BETWEEN WEIGHTED BANACH SPACES OF ANALYTIC FUNCTIONS
}

\author{
J. BONET, P. DOMAŃSKI, M. LINDSTRÖM and J. TASKINEN
}

(Received 19 February 1997)

Communicated by P. G. Dodds

\begin{abstract}
We characterize those analytic self-maps $\varphi$ of the unit disc which generate bounded or compact composition operators $C_{\varphi}$ between given weighted Banach spaces $H_{v}^{\infty}$ or $H_{v}^{0}$ of analytic functions with the weighted sup-norms. We characterize also those composition operators which are bounded or compact with respect to all reasonable weights $v$.
\end{abstract}

1991 Mathematics subject classification (Amer. Math. Soc.): primary 47B38; secondary 30D55, 46E15.

\section{Introduction}

The aim of this paper is to study boundedness and compactness of composition operators $C_{\varphi}, C_{\varphi}(f)=f \circ \varphi$ on weighted Banach spaces of analytic functions, where $\varphi: \mathbb{D} \rightarrow \mathbb{D}$ is an analytic map on the unit disc $\mathbb{D}$. We are interested in complex spaces of the form

$$
\begin{gathered}
H_{v}^{\infty}:=H_{v}^{\infty}(\mathbb{D}):=\left\{f \in H(\mathbb{D}):\|f\|_{v}:=\sup _{z \in \mathbb{D}} v(z)|f(z)|<\infty\right\}, \\
H_{v}^{0}:=H_{v}^{0}(\mathbb{D}):=\left\{f \in H(\mathbb{D}): \lim _{|z| \rightarrow 1^{-}} v(z)|f(z)|=0\right\},
\end{gathered}
$$

endowed with the norm $\|\cdot\|_{v}$, where $H(\mathbb{D})$ denotes the space of analytic functions on $\mathbb{D}$ and $v: \mathbb{D} \rightarrow \mathbb{R}_{+}$is an arbitrary weight, that is, bounded continuous positive (which means strictly positive throughout the paper) function.

The research of the first named author was partially supported by DGICYT, grant. no. PB94 - 0541. The research of the second named author was mainly done while he visited Universidad Politécnica de Valencia, Spain, supported by DGICYT (Spain), grant no. SAB 95-0092 in the academic year 1995/96. (C) 1998 Australian Mathematical Society $0263-6115 / 98 \$ A 2.00+0.00$ 
Composition operators were extensively studied on various 'integral type' spaces of analytic functions on the disc like Hardy spaces, Bergman spaces, Dirichlet spaces or Bloch spaces (see, for example, [CM, Sh] and $[\mathrm{J}]$ ) and weighted spaces of continuous functions (see [SS]). The spaces $H_{v}^{\infty}$ or $H_{v}^{0}$ are connected with the study of growth conditions of analytic functions and were also studied in detail (see [SW1, SW2, RS, BS, BBT, L1, L2]). Our purpose is to connect both topics.

Especially interesting are radial weights $v$, that is, $v(z)=v(|z|)$. In that case, if $v \equiv 1$, then we get $H_{v}^{\infty}=H^{\infty}$ the space of all bounded analytic functions and $H_{v}^{0}=\{0\}$. If limsup $\sup _{|=| \rightarrow 1^{-}} v(z)>0$, then obviously $H_{v}^{\alpha}=H^{x}$ with an equivalent norm and also $H_{v}^{0}=\{0\}$. Moreover, $C_{\varphi}: H^{\infty} \rightarrow H^{\infty}$ is always bounded and it is compact if and only if $\overline{\varphi(\mathbb{D})} \subseteq \mathbb{D}$ (see [CM, Ex. 3.3.2]). Now, if $\lim _{|=| \rightarrow 1^{-}} v(z)=0$, the so-called non-increasing majorant of $v$, that is, the radial function $u: \mathbb{D} \rightarrow \mathbb{R}_{+}$, $u(r)=\sup \{v(R): r \leq R<1\}$, is also continuous and tends to zero at the boundary. By the maximum principle $H_{u}^{\infty}=H_{v}^{\infty}, H_{u}^{0}=H_{v}^{0}$, and the corresponding spaces are isometric. On account of what was just said, we call any radial, positive continuous function $v: \mathbb{D} \rightarrow \mathbb{R}_{+}$, which is non-increasing with respect to $|z|$ and is such that $\lim _{|=| \rightarrow 1^{-}} v(z)=0$, a typical weight. We will be mostly interested in the radial weights but as we have seen we may assume that the weight is typical. Nevertheless, we formulate our results in a more general (even non-radial) setting whenever it is possible.

Let us explain the organization of the paper. In Section 1 we summarize preliminaries on spaces $H_{v}^{\infty}$ and $H_{v}^{0}$. In Section 2 we collect results on boundedness of $C_{\varphi}$ and in Section 3 analogous results on compactness. The last Section 4 contains some results on $p$-integral composition operators.

We finish the introduction with some notation. If $1 \leq p<\infty$ and $v$ is a weight, we define the Banach space

$$
A_{v}^{p}:=A_{v}^{p}(\mathbb{D}):=\left\{f \in H(\mathbb{D}):\|f\|^{p}:=\int_{=\in D}|f(z)|^{p} v(z)^{p} d A(z)<\infty\right\},
$$

where $d A$ is the 2 -dimensional Lebesgue measure. If $f: \mathbb{D} \rightarrow \mathbb{C}$ is an analytic function, then $M(f, r):=\sup _{|=|=r}|f(z)|$ is a log-convex non-decreasing function (see the Hadamard Three Circle Theorem [C, V.3.13]). We reserve the letters $v, w$ for weights. We denote the natural numbers by $\mathbb{N}=\{1,2,3 \ldots\}$. By $C, C^{\prime}, c$ etcetera we denote positive constants which may vary from place to place but do not depend on indices or variables in given formulas or inequalities.

\section{The spaces $H_{v}^{\infty}$ and $H_{v}^{0}$}

Notice that the norm topology on $H_{v}^{\infty}$ is stronger than the topology $\tau_{c o}$ of uniform convergence on the compact sets of $\mathbb{D}$. Assume for a while that $\lim _{|=| \rightarrow 1^{-}} v(z)=0$. 
Since $f_{r}(z):=f(r z)$ tends in $\tau_{c o}$ to $f$, the closed unit ball $B_{v}^{0}$ of $H_{v}^{0}$ is $\tau_{c o}$-dense in the unit ball $B_{v}$ of $H_{v}^{\infty}$. This implies (see [BS, Th. 1.1], compare [RS, Th. 1]) that $\left(H_{v}^{0}\right)^{\prime \prime}=$ $H_{\mathrm{r}}^{\infty}$ isometrically and the embedding of $H_{v}^{0}$ into $H_{v}^{\infty}$ is the canonical embedding into its bidual. Moreover, since point evaluations are continuous functionals on $H_{v}^{0}$, the pointwise convergence topology (denoted by $\tau_{p}$ ) on $H_{v}^{0}$ is weaker than the weak topology. Looking at the representation of $\left(H_{v}^{0}\right)^{\prime}$ (see [BS, Th. 1.1]), we realize immediately that if $\delta_{z}$ is a point evaluation at $z$ on $H_{v}^{0}$, then for $f \in H_{v}^{\infty}$ we have $\left\langle f, \delta_{z}\right\rangle=f(z)$. Thus the pointwise convergence on $H_{v}^{\infty}$ is weaker than its weak-star topology. Since $H_{r}^{0}$ is $\tau_{p}$-dense in $H_{v}^{\infty}$ and $C_{\varphi}$ is always $\tau_{p}$-continuous, $C_{\varphi}: H_{v}^{\infty} \rightarrow H_{w}^{\infty}$ is equal to the bi-adjoint map of $C_{\varphi}: H_{v}^{0} \rightarrow H_{w}^{0}$ whenever both operators are well defined and $\lim _{|=| \rightarrow 1^{-}} v(z)=\lim _{|=| \rightarrow 1^{-}} w(z)=0$.

In fact, from the papers of Lusky [L1, L2], we know that for radial weights and under quite general assumptions $H_{v}^{0} \simeq c_{0}$ and $H_{v}^{\infty} \simeq l_{\infty}$.

To each weight $v$ corresponds the so-called growth condition $u: \mathbb{D} \rightarrow \mathbb{R}_{+}, u=1 / v$ and $B_{v}=\{f \in H(\mathbb{D}):|f| \leq u\}$. In [BBT] the new function $\tilde{u}: \mathbb{D} \rightarrow \mathbb{R}_{+}$is defined by $\tilde{u}(z):=\sup _{f \in B_{u}}|f(z)|$ and the weight associated with $v$ is defined by $\tilde{v}:=1 / \tilde{u}$. It is shown there that $\tilde{u}$ and $\tilde{v}$ have the following useful properties:

(i) $0<\tilde{u} \leq u$ and $0<v \leq \tilde{v}, \tilde{v}$ is bounded;

(ii) $\tilde{u}$ and $\tilde{v}$ are continuous and, respectively, radial, non-decreasing and nonincreasing whenever $u$ and $v$ are so;

(iii) $\|f\|_{v} \leq 1$ if and only if $\|f\|_{\bar{v}} \leq 1$ for $f \in H(\mathbb{D})$;

(iv) for every $z \in \mathbb{D}$ there is $f_{z} \in B_{v}$ with $\tilde{u}(z)=\left|f_{z}(z)\right|$;

(v) if $\lim _{|=| \rightarrow 1^{-}} v(z)=0$, then $\tilde{u}(z)=\sup _{f \in B_{r}^{0}}|f(z)|$.

As in $[\mathrm{T}]$ a weight $v$ is called essential if there exists a $C>0$ such that $v(z) \leq$ $\tilde{v}(z) \leq C v(z)$ for all $z \in \mathbb{D}$.

There are many known criteria for $v$ to be essential (see [BBT], especially Proposition 3.4 there). In particular, if $v(z)=1 / M(f,|z|)$ for some analytic function $f: \mathbb{D} \rightarrow \mathbb{C}$, then $v=\tilde{v}$. It turns out that tending to zero at the boundary is preserved by the tilde operation.

PROPOSITION 1.1. Let $v$ be a weight on $\mathbb{D}$. Then $\lim _{|z| \rightarrow 1^{-}} \tilde{v}(z)=0$, whenever $\lim _{|=| \rightarrow 1} v(z)=0$. In particular, if $v$ is a typical weight, then $\tilde{v}$ is typical as well.

COROLLARY 1.2. If $\lambda: \mathbb{D} \rightarrow \mathbb{R}_{+}$is a radial weight then $H_{\lambda}^{\infty}$ is strictly bigger than $H^{\infty}$ if and only if $\lim _{|=| \rightarrow 1^{-}} \lambda(z)=0$.

ProOf OF PROPOSITION 1.1. Let us take $u=1 / v$ as usual; then the growth condition $u$ tends to $+\infty$ at the boundary. Since there is a radial non-decreasing function $\leq u$ tending to $+\infty$ at the boundary, we may (and we will) assume that $u$ is radial.

Let $r_{n} \in(0,1), u\left(r_{n}\right)=n$. We take $f_{0} \equiv 0$ and we define inductively a sequence of functions $\left(f_{n}\right)$ analytic on a neighbourhood of $\overline{\mathbb{D}}$ such that: 
(a) $M\left(f_{j}, r_{i}\right)<i$ for $i \leq j$;

(b) $M\left(f_{j}, 1\right)=j$ and $f_{j}(1)=j$;

(c) $M\left(f_{j}-f_{j-1}, r_{j}\right) \leq 2^{-j}$.

Assume that $f_{1}, \ldots, f_{n-1}$ are defined satisfying (a) - (c) for $j \leq n-1$. Then we define $b_{n}, 0<b_{n}<2^{-n}$, such that $b_{n}<i-M\left(f_{n-1}, r_{i}\right)$ for $i=1, \ldots, n$. We define a function $g$, analytic on a neighbourhood of $\overline{\mathbb{D}}$ such that $g(z):=a_{n} /\left(R_{n}-z\right)^{n}$, where

$$
a_{n}=\left(\frac{1-r_{n}}{b_{n}^{-1 / n}-1}\right)^{n}, \quad R_{n}=1+\frac{1-r_{n}}{b_{n}^{-1 / n}-1} .
$$

It is easily seen that:

$$
M\left(g, r_{n}\right) \leq b_{n}, \quad g(1)=1, \quad M(g, 1)=1 .
$$

By the choice of $b_{n}$ we obtain that $f_{n}=f_{n-1}+g$ satisfies conditions (a) - (c) for $j=n$. Let us define $f:=\lim _{n \rightarrow \infty} f_{n}$. Since $M\left(f, r_{n}\right) \leq n=u\left(r_{n}\right)$ for $n \in \mathbb{N}$, we have $|f(z)| \leq u(z)+1$ for any $z \in \mathbb{D}$ and there is a constant $C$ such that $|f(z)| \leq C u(z)$ for any $z \in \mathbb{D}$. Clearly, $\tilde{u}(z) \geq|f(z)| / C$ and $\lim _{\mid=1 \rightarrow 1^{-}} \tilde{u}(z)=+\infty$. This completes the proof.

In any case we can substitute $v$ by $\tilde{v}$ but unfortunately we have no easy way of calculating $\tilde{v}$ from $v$.

PROPOSITION 1.3. For every weight $v$ we have isometrically $H_{v}^{\infty}=H_{\tilde{v}}^{\infty}$ and, if $\lim _{|z| \rightarrow 1^{-}} v(z)=0$, then $H_{v}^{0}=H_{\tilde{v}}^{0}$.

PROOF. By the property (iii), $H_{v}^{\infty}=H_{\tilde{v}}^{\infty}$ isometrically and $H_{\tilde{v}}^{0}$ is an isometric (closed) subspace of $H_{v}^{0}$. As we mentioned before, if $T=C_{\text {id }}: H_{v}^{0} \rightarrow H_{v}^{0}$, then $T^{\prime \prime}: H_{\tilde{v}}^{\infty} \rightarrow H_{v}^{\infty}$ is also equal to $C_{\text {id }}: H_{\tilde{v}}^{\infty} \rightarrow H_{v}^{\infty}$. Clearly, if $T$ were not onto, then $T^{\prime \prime}$ would have not been onto as well.

\section{Boundedness of $C_{\varphi}$}

We give firstly necessary and sufficient conditions for the operator $C_{\varphi}$ to be bounded on $H_{v}^{\infty}$.

PROPOSITION 2.1. Let $v$ and $w$ be weights. The following statements are equivalent:

(i) the operator $C_{\varphi}: H_{v}^{\infty} \rightarrow H_{w}^{\infty}$ is bounded;

(ii) $\sup _{z \in \mathbb{D}} w(z) / \tilde{v}(\varphi(z))=M<\infty$, 
(iii) $\sup _{z \in \mathbb{D}} \tilde{w}(z) / \tilde{v}(\varphi(z))=M<\infty$.

If $v$ and $w$ satisfy $\lim _{|z| \rightarrow 1^{-}} v(z)=\lim _{|z| \rightarrow 1^{-}} w(z)=0$, then the above conditions are equivalent to

(iv) the operator $C_{\varphi}: H_{v}^{0} \rightarrow H_{w}^{0}$ is bounded.

REMARK. Contrary to many cases of classical function spaces, an analytic self-map $\varphi: \mathbb{D} \rightarrow \mathbb{D}$ does not necessarily induce a bounded composition operator for general weights. For example, consider $v(z)=w(z)=e^{-(1-|z|)^{-1}}$ and $\varphi(z)=(z+1) / 2$. Then $v=\tilde{v}$ and, for $z=r \in \mathbb{R}, v(r) / v(\varphi(r))=e^{1 /(1-r)}$ for $0<r<1$. Thus $v(r) / v(\varphi(r)) \rightarrow \infty$, when $r \rightarrow 1$, so $C_{\varphi}$ is not bounded on $H_{v}^{\infty}$.

If $v$ is essential, then for every $z \in \mathbb{D}$ we have that $\tilde{v}(z) \leq C v(z)$, and the necessity of (4) in the next corollary follows from this.

COROLlARY 2.2. Assume that $v$ is an essential weight. The operator $C_{\varphi}: H_{v}^{\infty} \rightarrow$ $H_{u}^{\infty}$ is bounded if and only if

$$
\sup _{z \in \mathbb{D}} w(z) / v(\varphi(z))<\infty
$$

If $v$ and $w$ satisfy $\lim _{|z| \rightarrow 1^{-}} v(z)=\lim _{|z| \rightarrow 1^{-}} w(z)=0$, then $C_{\varphi}: H_{v}^{0} \rightarrow H_{w}^{0}$ is bounded if and only if (4) holds.

REMARKS. (1) If $H_{v}^{\infty}=H^{\infty}$, then $C_{\varphi}: H_{v}^{\infty} \rightarrow H_{w}^{\infty}$ is continuous for all weights $w$ and, if $w$ tends to zero at the boundary $C_{\varphi}\left(H_{v}^{\infty}\right) \subseteq H_{w}^{0}$. On the other hand, by Proposition 1.1 and by properties of associated weights, one can prove that if $\left(z_{n}\right) \subseteq \mathbb{D}$, $\lim _{n \rightarrow \infty}\left|z_{n}\right|=1$ and $v$ is a weight on $\mathbb{D}$ tending to zero at the boundary, then there is a sequence of functions $f_{n} \in B_{v}$ such that $\left|f_{n}\left(z_{n}\right)\right| \rightarrow \infty$ as $n \rightarrow \infty$. Thus, if $H_{w}^{\infty}=H^{\infty}$ and $\partial \varphi(\mathbb{D}) \cap \partial \mathbb{D} \neq \emptyset$ then $C_{\varphi} f_{n}$ is unbounded in $H_{w}^{\infty}$. Finally, in that case $C_{\varphi}: H_{v}^{\infty} \rightarrow H_{w}^{\infty}$ is bounded if and only if $\overline{\varphi(\mathbb{D})} \subseteq \mathbb{D}$.

(2) The condition in the corollary is no more necessary for boundedness of $C_{\varphi}$ whenever $v$ is not essential. Let us take an arbitrary non-essential weight $v$ and $w=\tilde{v}$. Then, clearly $\lim \sup _{|z| \rightarrow 1^{-}} w(z) / v(z)=+\infty$ but $C_{\text {id }}: H_{v}^{\infty} \rightarrow H_{w}^{\infty}$ is an isometry.

PROOF OF PROPOSITION 2.1. (iii) implies (ii) is trivial as $w \leq \tilde{w}$.

(ii) implies (i): By assumption, we have $w(z) \leq M \tilde{v}(\varphi(z))$ for all $z \in \mathbb{D}$. Thus

$$
w(z)|f(\varphi(z))|=\frac{w(z)}{\tilde{v}(\varphi(z))} \tilde{v}(\varphi(z))|f(\varphi(z))| \leq M\|f\|_{\tilde{v}}
$$

(i) implies (iii) and (iv) implies (iii): If not, then there is a sequence $\left(z_{n}\right) \subset \mathbb{D}$, with $\tilde{w}\left(z_{n}\right)>n \tilde{v}\left(\varphi\left(z_{n}\right)\right)$ for all $n$. For all $n$, there exists $f_{n} \in B_{v}$ (which can be chosen in 
$B_{v}^{0}$, whenever $\lim _{|=| \rightarrow 1^{-}} v(z)=0$, by $\tau_{p}$-density of $B_{v}^{0}$ in $\left.B_{v}\right)$ such that $\left|f_{n}\left(\varphi\left(z_{n}\right)\right)\right|>$ $\tilde{u}\left(\varphi\left(z_{n}\right)\right) / 2$. By (i) or (iv), $\left(f_{n} \circ \varphi\right)$ is bounded in $H_{u^{\prime}}^{\infty}(D)=H_{\tilde{u}^{\prime}}^{\infty}(D)$, so there is $C>0$ such that $\left|f_{n}(\varphi(z))\right| \tilde{w}(z) \leq C$ for all $z \in D$ and all $n \in \mathbb{N}$. On the other hand,

$$
\left|f_{n}\left(\varphi\left(z_{n}\right)\right)\right| \tilde{w}\left(z_{n}\right)=\left|f_{n}\left(\varphi\left(z_{n}\right)\right)\right| \tilde{v}\left(\varphi\left(z_{n}\right)\right) \tilde{w}\left(z_{n}\right) / \tilde{v}\left(\varphi\left(z_{n}\right)\right)>n / 2
$$

for all $n$, so we have a contradiction.

(iii) implies (iv): By (iii) implies (i) and Proposition 1.3, it suffices to show that $C_{\varphi}(f) \in H_{\tilde{w}}^{0}$ for each $f \in H_{\tilde{v}}^{0}$.

Take $f \in H_{\tilde{v}}^{0}$. Given $\varepsilon>0$, there is $\left.r_{1} \in\right] 0,1[$, such that $\tilde{v}(z)|f(z)|<\varepsilon / M$ for $|z|>r_{1}$. For $|z|>r_{1}$ we consider two cases: If $|\varphi(z)|>r_{1}$, then

$$
\tilde{w}(z)|f(\varphi(z))|=\tilde{v}(\varphi(z))|f(\varphi(z))| \frac{\tilde{w}(z)}{\tilde{v}(\varphi(z))}<\varepsilon .
$$

For $|\varphi(z)| \leq r_{1}$, we have that there is $r_{2} \geq r_{1}, 0<r_{2}<1$, such that

$$
\tilde{w}(z)|f(\varphi(z))| \leq \tilde{w}(z) \sup _{|=| \leq r_{1}}|f(z)|<\varepsilon \text { for }|z|>r_{2} .
$$

Thus $\tilde{w}(z)|f(\varphi(z))|<\varepsilon$ for $|z|>r_{2}$.

THEOREM 2.3. Let $v$ be a typical weight. The following assertions are equivalent:

(i) all operators $C_{\varphi}: H_{v}^{\infty} \rightarrow H_{v}^{\infty}$ are bounded;

(ii) all operators $C_{\varphi}: H_{v}^{0} \rightarrow H_{v}^{0}$ are bounded;

(iii) the following inequality holds:

$$
\inf _{n \in \mathbb{N}} \frac{\tilde{v}\left(1-2^{-n-1}\right)}{\tilde{v}\left(1-2^{-n}\right)}>0 .
$$

REMARK. For example, an essential weight $v(z)=(1-|z|)^{\alpha}, \alpha>0$, satisfies the conditions of Theorem 2.3 The condition (5) was used by Lusky [L2, p. 310], when he studied the isomorphism $H_{v}^{0} \simeq c_{0}$. Also see [SW2].

PROOF. By Proposition 2.1, (i) holds if and only if (ii) does.

By the Schwarz lemma, if $\varphi(0)=0$, then $|\varphi(z)| \leq|z|$ for every $z \in \mathbb{D}$ and therefore $C_{\varphi}: H_{v}^{\infty} \rightarrow H_{v}^{\infty}$ is bounded. For $p \in \mathbb{D}$, let

$$
\alpha_{p}(z)=(p-z) /(1-\bar{p} z),
$$

that takes $\mathbb{D}$ onto itself. If each $C_{\alpha_{p}}$ is bounded, then all $C_{\psi}$ are bounded. Indeed, each $\varphi=\alpha_{p} \circ \psi$, where $\psi=\alpha_{p} \circ \varphi, p=\varphi(0)$ and $\psi(0)=0$. We have to show that for any $p \in \mathbb{D}, C_{\alpha_{p}}$ is bounded on $H_{v}^{\infty}(\mathbb{D})$ if and only if $\tilde{v}$ satisfies (5). 
(i) implies (iii): Let us assume that all $C_{\alpha_{\rho}}$ are bounded. Then, by Proposition 2.1, for every $p \in \mathbb{D}$ there exist $M_{p}>0$ such that $\tilde{v}(z)<M_{p} \tilde{v}\left(\alpha_{p}(z)\right)$ for all $z \in \mathbb{D}$. Since it is easily seen that $\sup _{|=|=r}\left|\alpha_{p}(z)\right|=(|p|+r) /(1+|p| r)$, we get that $\tilde{v}(z)<$ $M_{p} \tilde{v}((|p|+r) /(1+|p| r))$ for all $|z|=r$. Let us define $l(r)=\tilde{v}(1-r), s=1-r$. Since $1-(|p|+1-s) /(1+|p|(1-s))=s(1-|p|) /(1+|p|-|p| s)$, we obtain for $s<1 / 2$ that

$$
l\left(s \frac{1-|p|}{1+|p|}\right) \leq l\left(1-\frac{|p|+1-s}{1+|p|(1-s)}\right) \leq l\left(s \frac{(1-|p|)}{1+|p| / 2}\right) .
$$

Finally, for $p=2 / 5$, we use the second inequality in (6) to get $M>0$ and $s_{0}>0$ such that $l(s) \leq M l\left(\frac{s}{2}\right)$ for all $\left.s \in\right] 0, s_{0}[$. This immediately implies (5).

(iii) implies (i): If (5) is satisfied, then $l$ defined as above has the property that there are $M>0$ and $\left.t_{0} \in\right] 0,1\left[\right.$ with $l(t) \leq M l(t / 2)$ for all $t>t_{0}$. Then for any $c<\infty$ we find $n \in \mathbb{N}$ such that $c<2^{n}$ and hence $l(t) \leq M^{n} l\left(\frac{t}{c}\right)$. We take $c=(1+|p|) /(1-|p|)$. Then by the first inequality in (6), for all $p \in \mathbb{D}$ there is $M_{p}>0$ with

$$
l(t) \leq M_{p} l\left(1-\frac{|p|+1-t}{1+|p|(1-t)}\right),
$$

for all $t>t_{0}$. Clearly, this implies that for all $p \in \mathbb{D}$ there exists $M_{p}>0$ such that for every $|z|=r$ we have that $\tilde{v}(z)<M_{p} \tilde{v}\left(\alpha_{p}(z)\right)$ by the argument above.

THEOREM 2.4. Let $\varphi: \mathbb{D} \rightarrow \mathbb{D}$ be an analytic map. The following statements are equivalent:

(i) for any typical weight $v$ the map $C_{\varphi}$ is bounded on $H_{v}^{\infty}$;

(ii) for every $\theta \in]-\pi, \pi], \alpha_{\theta} \circ \varphi$ fixes a point in $\mathbb{D}$, where $\alpha_{\theta}(z)=e^{i \theta} z$;

(iii) either $\varphi$ is a rotation or for any $\theta \in]-\pi, \pi], \psi=\alpha_{\theta} \circ \varphi$ fixes an attracting point $p_{\theta} \in \mathbb{D}$, that is, $\psi\left(p_{\theta}\right)=p_{\theta}$ and $\psi_{n}(z) \rightarrow p_{\theta}$ uniformly on compact sets, where $\psi_{n}:=\psi \circ \cdots \circ \psi,(n$ times $)$

(iv) either $\varphi$ is a rotation or $\liminf _{|=| \rightarrow 1^{-}}(1-|\varphi(z)|) /(1-|z|)>1$;

(v) there is an $\left.r_{0} \in\right] 0,1\left[\right.$ such that $|\varphi(z)| \leq|z|$ for every $z \in \mathbb{D}$ with $|z| \geq r_{0}$.

For the proof of Theorem 2.4 we need the following result.

LEMMA 2.5. For any two increasing sequences $r_{n} \rightarrow 1$ and $R_{n} \rightarrow 1$ such that $r_{0}<R_{0}<r_{1}<R_{1}<r_{3}<R_{3}<\cdots$ there is an analytic map $f: \mathbb{D} \rightarrow \mathbb{C}$ with

$$
\lim _{n \rightarrow \infty} \frac{M\left(f, R_{n}\right)}{M\left(f, r_{n}\right)}=\infty
$$

where $M(f, r)=\sup _{|z|=\mid}|f(z)|$. 
ProOF. We define $f_{0} \equiv 1$. Assume that we have already found polynomials $f_{1}, f_{2}, \ldots, f_{n-1}$ such that

(a) $\left.f_{k}\right|_{r_{k} \mathbb{D}}<1 / 2^{k}$ (b) $\frac{M\left(f_{k}, R_{k}\right)}{M\left(\sum_{i=1}^{k-1} f_{i}, r_{k}\right)}>k$ for $k=1, \ldots, n-1$.

Then put $M:=M\left(\sum_{i=1}^{n-1} f_{i}, r_{n}\right)$ and $\tilde{f}_{n}(z)=A /\left((1-\varepsilon) R_{n}-z\right)$, where $A, \varepsilon$ are chosen positive and
(c)
$\frac{A}{(1-\varepsilon) R_{n}-r_{n}}=\frac{1}{2^{2 n}}$
(d) $\frac{A}{\varepsilon R_{n}}=2 M n$.

In fact, it suffices to take

$$
A=\frac{2 M n\left(R_{n}-r_{n}\right)}{\left(2^{2 n+1} M n+1\right)} \quad \text { and } \quad \varepsilon=\frac{\left(R_{n}-r_{n}\right)}{R_{n}\left(2^{2 n+1} M n+1\right)}
$$

Clearly $\left.\tilde{f}_{n}\right|_{r_{n} \mathbb{D}}<1 / 2^{2 n}$ because of (c). Moreover, by $(\mathrm{d}), \tilde{f}_{n}\left(R_{n}\right)=2 n M$. Now, by Runge's Theorem, we can approximate $\tilde{f}_{n}$ on $\overline{R_{n} \mathbb{D}}$ by a polynomial $f_{n}$ satisfying (a) and (b). We define $f=\sum_{i=1}^{\infty} f_{i}$. The series converges almost uniformly. The condition $\lim _{n} M\left(f, R_{n}\right) / M\left(f, r_{n}\right)=\infty$ follows from (b).

PROOF OF THEOREM 2.4. (i) implies (v): Assume that there is a sequence $\left(z_{n}\right)$ in $\mathbb{D}$ with $\left|z_{n}\right| \rightarrow 1$ such that $\left|\varphi\left(z_{n}\right)\right|>\left|z_{n}\right|$ for all $n$. Define $r_{n}=\left|z_{n}\right|, R_{n}=\left|\varphi\left(z_{n}\right)\right|$ and without loss of generality we may assume that $r_{0}<R_{0}<r_{1}<R_{1}<r_{2}<R_{2}<\cdots$. By Lemma 2.5, there exists $f \in H(\mathbb{D})$ such that $\lim _{n \rightarrow \infty} M\left(f, R_{n}\right) / M\left(f, r_{n}\right)=\infty$. Take $v(z)=1 / M(f,|z|)$ and assume that $C_{\varphi}: H_{v}^{\infty} \rightarrow H_{v}^{\infty}$ is bounded. Then there is $c>0$ such that $\left\|C_{\varphi}\right\| \leq c$. Moreover, with $\alpha_{\theta}(z)=e^{i \theta} z,\left\|C_{\alpha_{\theta}}\right\|=1$ and $\|f\|_{v}=1$, so

$$
\left.\left.\left\|C_{\alpha_{\theta} \circ \varphi}(f)\right\|_{v} \leq c \quad \text { for all } \quad \theta \in\right]-\pi, \pi\right]
$$

Now,

$$
\sup _{z \in D}\left|f \circ \alpha_{\theta} \circ \varphi(z)\right| \frac{1}{M(f,|z|)} \geq\left|f \circ \alpha_{\theta} \circ \varphi\left(z_{n}\right)\right| \frac{1}{M\left(f,\left|z_{n}\right|\right)}
$$

for all $n$ and all $\theta$. Choosing $\theta_{n}$ in a suitable way, we get $\left|f \circ \alpha_{\theta_{n}} \circ \varphi\left(z_{n}\right)\right|=$ $M\left(f,\left|\varphi\left(z_{n}\right)\right|\right)=M\left(f, R_{n}\right)$. Thus, $\left\|C_{\alpha_{\theta_{n}} \circ \varphi}(f)\right\|_{v} \geq M\left(f, R_{n}\right) / M\left(f, r_{n}\right)$ for all $n$, and we have a contradiction.

(v) implies (i) is obvious. 
(i) implies (ii): Let us assume firstly that $\varphi$ is an automorphism. Then either $\varphi$ is a rotation and (ii) is satisfied or $\varphi(z)=\alpha_{\theta}((p-z) /(1-\bar{p} z))$, where $p \neq 0$. Since $\sup _{|z|=r}|\varphi(z)|=(|p|+r) /(1+|p| r)>r$ for $r<1$, it follows that (v) is not satisfied. We have proved that if $\varphi$ is an automorphism, then $C_{\varphi}$ satisfies (i) only for rotations, and then (ii) is satisfied as well.

Now let $\varphi$ be a non-automorphism. Then every $\alpha_{\theta} \circ \varphi$ is also a non-automorphism. Hence, by [Sh, Section 5.4], either $\alpha_{\theta} \circ \varphi$ has a fixed point or $\psi_{n}:=\psi \circ \cdots \circ \psi,(n$ times), where $\psi=\alpha_{\theta} \circ \varphi$, satisfies $\left|\psi_{n}(z)\right| \rightarrow 1$ uniformly on compact sets. Suppose that some $\alpha_{\theta} \circ \varphi$ has no fixed point. Let $K:=\left\{z:|z| \leq r_{0}\right\}$, where $r_{0}$ is given in $(v)$. By the Maximum Modulus Theorem, $\sup _{z \in K}|\varphi(z)|=\sup _{z \in \partial K}|\varphi(z)|$ and therefore it follows from $(v)$ that $\sup _{=\in \partial K}\left|\psi_{n}(z)\right| \leq r_{0}$. Since $\left|\psi_{n}(z)\right| \rightarrow 1$ uniformly on compact sets, we obtain a contradiction.

(ii) implies (iii): Assume that $\varphi$ is an automorphism. Hence

$$
\left.\left.\varphi(z)=e^{i \theta} \frac{p-z}{1-\bar{p} z} \quad \text { for some } \quad p \in \mathbb{D}, \theta \in\right]-\pi, \pi\right] .
$$

If $p \neq 0$, then for a suitable chosen $\theta_{0},|p|=\cos \left(\theta_{0} / 2\right)$ and by [Sh, Section 0.5.4], $\varphi$ is a parabolic automorphism, meaning (see [Sh, p. 5]) that $\varphi$ has a single fixed point lying on the boundary of $\mathbb{D}$. If $p=0$, then $\varphi$ is a rotation.

Now, let $\varphi$ be a non-automorphism. Then every $\alpha_{\theta} \circ \varphi$ is also a non-automorphism. Hence the assumption and [Sh, Section 5.2.1], give that every $\alpha_{\theta} \circ \varphi$ fixes an attracting point in $\mathbb{D}$.

(iii) implies (iv): Let us assume that each $\alpha_{\theta} \circ \varphi$ has an attracting fixed point $p_{\theta}$ in $\mathbb{D}$. If $\lim \inf _{|=| \rightarrow 1^{-}}(1-|\varphi(z)|) /(1-|z|) \leq 1$, then there is a sequence $\left(z_{n}\right) \subset \mathbb{D},\left|z_{n}\right| \rightarrow 1$ and $\lim _{n}\left(1-\left|\varphi\left(z_{n}\right)\right|\right) /\left(1-\left|z_{n}\right|\right)=\delta \leq 1$. Without loss of generality, we may assume that $z_{n} \rightarrow w \in \partial \mathbb{D}$. Clearly, $\left|\varphi\left(z_{n}\right)\right| \rightarrow 1$, and we may assume that $\varphi\left(z_{n}\right) \rightarrow \eta \in \partial \mathbb{D}$. Choosing $\theta \in]-\pi, \pi$ ] suitable, we get $\alpha_{\theta} \circ \varphi\left(z_{n}\right) \rightarrow w$. Now, by Julia's theorem [Sh, p. 63],

$$
\alpha_{\theta} \circ \varphi\left(\frac{\lambda}{1+\lambda} \mathbb{D}+\frac{w}{1+\lambda}\right) \subset \frac{\lambda}{1+\lambda} \mathbb{D}+\frac{w}{1+\lambda} \quad \text { for all } \quad \lambda>0 .
$$

Since $p_{\theta}$ is an attracting point of $\alpha_{\theta} \circ \varphi$, it is in any disc $(\lambda /(1+\lambda)) \mathbb{D}+w /(1+\lambda)$, which gives a contradiction by taking $\lambda>0$ small enough.

(iv) implies (v): The condition is obviously satisfied for rotations. Now, if $\liminf _{|z| \rightarrow 1^{-}}(1-|\varphi(z)|) /(1-|z|)>1$, then there is $\left.r_{0} \in\right] 0,1[$ such that $(1-|\varphi(z)|) /$ $(1-|z|)>1$ for $|z|>r_{0}$. Clearly, then $|\varphi(z)|<|z|$, and we are done. 


\section{Compactness of $C_{\varphi}$}

To deal with the compactness we need the following form of the Weak Compactness Theorem. The proof is similar to the case of $H^{2}$. The reader is asked to refer to [Sh, Section 2.4].

LEMMA 3.1. Let $X$ and $Y$ be $H_{v}^{\infty}$ and $H_{u}^{\infty}$ or $H_{r}^{0}$ and $H_{u}^{(1)}$ respectively. A bounded operator $C_{\varphi}: X \rightarrow Y$ is compact, if and only if, given any bounded sequence $\left(f_{n}\right) \subset X$ which converges to 0 uniformly on the compact subsets of $\mathbb{D}$, also the sequence $\left(C_{\varphi}\left(f_{n}\right)\right)$ converges to 0 in $Y$.

COROLLARY 3.2. Let $v$ and $w$ be weights. If there exists an $r, 0<r<1$, such that $|\varphi(z)| \leq r$ for all $z \in \mathbb{D}$, then $C_{\varphi}: H_{v}^{\infty} \rightarrow H_{u^{\prime}}^{\infty}$ is compact.

THEOREM 3.3. Let $v$ and $w$ be weights. The following assertions are equivalent:

(i) the operator $C_{\varphi}: H_{v}^{\infty} \rightarrow H_{w}^{\infty}$ is compact;

(ii) $\lim _{r \rightarrow 1^{-}} \sup _{|\varphi(z)|>r} w(z) / \tilde{v}(\varphi(z))=0 \operatorname{or} \overline{\varphi(\mathbb{D})} \subseteq \mathbb{D}$.

If $v$ and $w$ satisfy $\lim _{|=| \rightarrow 1^{-}} v(z)=\lim _{|=| \rightarrow 1^{-}} w(z)=0$, then the above conditions are also equivalent to the following ones:

(iii) the operator $C_{\varphi}: H_{v}^{0} \rightarrow H_{w}^{0}$ is compact;

(iv) $\lim _{|z| \rightarrow 1^{-}} w(z) / \tilde{v}(\varphi(z))=0$,

(v) $\lim _{|z| \rightarrow 1^{-}} \tilde{w}(z) / \tilde{v}(\varphi(z))=0$.

REMARKS. (1) It is not difficult to show that if $H_{v}^{\infty}=H^{\infty}$, then $C_{\varphi}: H_{v} \times H_{u}^{\infty}$ is compact for any weight $w$ tending to zero at the boundary. On the other hand, if $H_{w}^{\infty}=H^{\infty}$, then $C_{\varphi}: H_{v}^{\infty} \rightarrow H_{w}^{\infty}$ is compact if and only if $\overline{\varphi(\mathbb{D})} \subseteq \mathbb{D}$ (compare the remark after Proposition 2.1).

(2) If for a fixed typical weight $v, C_{\varphi}: H_{v}^{\infty} \rightarrow H_{v}^{\infty}$ is compact, then there is $r<1$ such that $|\varphi(z)|<|z|$ for $|z|>r$. Thus $C_{\varphi}: H_{w}^{\infty} \rightarrow H_{w}^{\infty}$ is bounded for any typical weight $w$.

(3) If for some weights $v, w$ tending to zero at the boundary, $C_{\varphi}: H_{v}{ }^{x} \rightarrow H_{u}{ }^{x}$ is compact, then it is weakly compact and $C_{\varphi}: H_{v}^{\infty} \rightarrow H_{v}^{0}$.

ProOF. We assume first that $v$ and $w$ satisfy $\lim _{|=| \rightarrow 1^{-}} v(z)=\lim _{|=| \rightarrow 1^{-}} w(z)=0$. (v) implies (iv) is obvious.

(iv) implies (i): By Proposition 2.1, $C_{\varphi}$ is bounded. We shall use Lemma 3.1. Take a bounded sequence $\left(f_{n}\right) \subset B_{v}$ and assume that $f_{n} \rightarrow 0$ in the $\tau_{c o}$-topology. For given $\varepsilon>0$ there is $\left.r_{0} \in\right] 0,1\left[\right.$ with $w(z)<\varepsilon \tilde{v}(\varphi(z)) / 2$ for all $|z|>r_{0}$. Put 
$C^{\prime}:=\sup _{z \in \mathbb{D}} w(z)<\infty$. For $n$ big enough, we have that $\sup _{|=| \leq r_{0}}\left|f_{n}(\varphi(z))\right| C^{\prime}<\varepsilon / 2$ and thus

$$
\begin{aligned}
\left\|C_{\varphi}\left(f_{n}\right)\right\|_{w^{\prime}} & \leq \sup _{|z| \leq r_{0}}\left|f_{n}(\varphi(z))\right| w(z)+\sup _{|=|>r_{0}}\left|f_{n}(\varphi(z))\right| w(z) \\
& \leq \frac{1}{2} \varepsilon+\frac{1}{2} \varepsilon\left\|f_{n}\right\|_{\tilde{v}} \leq \varepsilon,
\end{aligned}
$$

so (i) follows.

(i) implies (v): If not, then there are $c>0$ and a sequence $\left(z_{n}\right) \subset \mathbb{D},\left|z_{n}\right| \rightarrow 1$, with $\tilde{w}\left(z_{n}\right)>c \tilde{v}\left(\varphi\left(z_{n}\right)\right)$ for all $n$. For all $n$, there exists $f_{n} \in B_{v}$ such that $\left|f_{n}\left(\varphi\left(z_{n}\right)\right)\right|=$ $\tilde{u}\left(\varphi\left(z_{n}\right)\right)$. By going to a subsequence we can assume that $\varphi\left(z_{n}\right) \rightarrow z_{0}$ for some $z_{0} \in \overline{\mathbb{D}}$, when $n \rightarrow \infty$. If $\left|z_{0}\right| \neq 1$, then by assumption,

$$
0=\lim _{n} \tilde{w}\left(z_{n}\right) \geq c \lim _{n} \tilde{v}\left(\varphi\left(z_{n}\right)\right)=c \tilde{v}\left(z_{0}\right)>0,
$$

which is a contradiction. Thus $\left|z_{0}\right|=1$. Now, since $\left|\varphi\left(z_{n}\right)\right| \rightarrow 1$, there exist natural numbers $\alpha(n)$ with $\lim _{n} \alpha(n)=\infty$ and such that $\left|\varphi\left(z_{n}\right)\right|^{\alpha(n)} \geq 1 / 2$ for all $n$. We define the analytic functions $g_{n}(z):=z^{\alpha(n)} f_{n}(z)$ for all $n$. Clearly $\left(g_{n}\right)$ is a bounded sequence in $H_{v}{ }^{\infty}$. It converges pointwise to 0 because of the factor $z^{\alpha(n)}$. Hence, supposing that $C_{\varphi}$ is compact, Lemma 3.1 implies that $\left\|C_{\varphi}\left(g_{n}\right)\right\|_{w} \rightarrow 0$ as $n \rightarrow \infty$. On the other hand, we get for all $n$,

$$
\begin{aligned}
\left\|C_{\varphi}\left(g_{n}\right)\right\|_{\tilde{n}} & \geq\left|g_{n}\left(\varphi\left(z_{n}\right)\right)\right| \tilde{w}\left(z_{n}\right)=\left|\varphi\left(z_{n}\right)\right|^{\alpha(n)}\left|f_{n}\left(\varphi\left(z_{n}\right)\right)\right| \tilde{w}\left(z_{n}\right) \\
& \geq \frac{1}{2}\left|f_{n}\left(\varphi\left(z_{n}\right)\right)\right| c \tilde{v}\left(\varphi\left(z_{n}\right)\right) \geq \frac{1}{2} c,
\end{aligned}
$$

which is a contradiction.

(i) if and only if (iii): By Proposition 2.1, if one of the considered operators is compact, then both are bounded and $C_{\varphi}: H_{v}^{\infty} \rightarrow H_{w}^{\infty}$ is the bi-adjoint of $C_{\varphi}: H_{v}^{0} \rightarrow H_{w}^{0}$ (see Section 1). Apply the Schauder Theorem.

Now, in the general case, (i) implies (ii) is similar to (i) $\Rightarrow(v)$ and (ii) implies (i) is similar to (iv) implies (i).

COROLLARY 3.4. Let $v$ and $w$ be essential weights and assume that $\lim _{|=| \rightarrow 1} w(z)=$ 0 . Then $C_{\varphi}: H_{v}^{\infty} \rightarrow H_{u^{\prime}}^{\infty}$ is compact (or, equivalently, $C_{\varphi}: H_{v}^{0} \rightarrow H_{w}^{0}$ is compact), if and only if

$$
\lim _{|z| \rightarrow 1^{-}} \frac{w(z)}{v(\varphi(z))}=0
$$

We see below that for general weights our simple characterization of compactness in 3.4 fails. Of course, in that case $v$ is no more essential and this fact gives another motivation for the concept of an essential weight. 
EXAMPLE 3.5. There exist a typical weight $w$ and a compact composition operator $C_{\varphi}: H_{w}^{\infty} \rightarrow H_{w}^{\infty}$ which does not satisfy (7).

CONSTRUCTION. Let us define increasing sequences $\left(r_{n}\right),\left(p_{n}\right)$ tending to 1 as follows:

$$
r_{0}=\frac{1}{2}, \quad r_{n+1}=\frac{2 r_{n}}{1+r_{n}}, \quad p_{0}=0, \quad p_{n}<r_{n}<p_{n+1} \quad \text { for } n \in \mathbb{N} .
$$

Choose an increasing sequence $\left(a_{n}\right)$ of natural numbers such that

$$
a_{n}\left(\log r_{n+1}-\log r_{n}\right) \geq n
$$

We define three non-decreasing, unbounded and continuous functions $\eta, \theta, \tilde{\theta}: \mathbb{R}_{-} \rightarrow$ $\mathbb{R}_{+}$as follows:

(i) $\eta \equiv 0$ on $\left(-\infty, \log r_{0}\right]$ and for each $n \in \mathbb{N}$ the function $\eta$ is affine on $\left[\log r_{n}, \log r_{n+1}\right]$ with the derivative $\equiv a_{n}$;

(ii) for $n$ even $\theta \equiv \tilde{\theta} \equiv \eta$ on $\left[\log p_{n}, \log p_{n+1}\right]$;

(iii) for $n$ odd $\tilde{\theta}$ is affine on $\left[\log p_{n}, \log p_{n+1}\right]$;

(iv) for $n \operatorname{odd} \theta$ is affine on $\left[\log p_{n}, \log r_{n}\right]$ and constant on $\left[\log r_{n}, \log p_{n+1}\right]$.

We could choose $\left(p_{n}\right)$ in such a way that:

(v) $\left|\eta\left(\log p_{n}\right)-\eta\left(\log r_{n}\right)\right| \leq 1$ and $|\tilde{\theta}(s)-\eta(s)| \leq 1$ for all $s \in \mathbb{R}$.

Now, we can define our typical weights:

$$
v(z):=e^{-\eta(\log |z|)}, \quad w(z):=e^{-H(\log |z|)} .
$$

Clearly, our assumptions imply that $\theta(s) \geq \tilde{\theta}(s) \geq \eta(s)$ for $s \in \mathbb{R}_{-}$. By the Hadamard Three Circle Theorem and (v),

$$
\tilde{w}(z) \geq e^{-\tilde{\theta}(\log |z|)} \geq e^{-1} v(z) .
$$

On the other hand, on the annulus $\left\{z: r_{n} \leq|z| \leq r_{n+1}\right\}$, we have $v(z)=b_{n}|z|^{-a_{n}}$ with $a_{n}$ natural. Moreover, $1 / v(z)$ is a supremum of a sequence $M\left(b_{n}^{-1} z^{a_{n}},|z|\right)$, which means that $v=\tilde{v}$. Taking the above into account, we obtain that $\tilde{w}$ is equivalent to $v=\tilde{v}$.

We will show that $C_{\varphi}: H_{v}^{\infty}=H_{w}^{\infty} \rightarrow H_{w}^{\infty}=H_{v}^{\infty}$ is compact for $\varphi(z):=z /(2-z)$ but still $\lim _{|z| \rightarrow 1^{-}} w(z) / w(\varphi(z)) \neq 0$.

Observe, that $|\varphi(z)| \leq \varphi(|z|)=|z| /(2-|z|)$, thus if $|z| \in\left(r_{n}, r_{n+1}\right)$, then $|\varphi(z)| \leq$ $\varphi(|z|) \in\left(r_{n-1}, r_{n}\right)$. Hence

$$
\frac{v(z)}{v(\varphi(z))} \leq \frac{v(z)}{v(\varphi(|z|))}=C \frac{(2-|z|)^{a_{n-1}}}{|z|^{a_{n+1}-a_{n}}}
$$


and the function on the right hand side is decreasing with respect to $|z|$. Summarizing, by (8) for $|z| \in\left(r_{n}, r_{n+1}\right)$,

$$
\frac{v(z)}{v(\varphi(z))} \leq \frac{v\left(r_{n}\right)}{v\left(\varphi\left(r_{n}\right)\right)}=\frac{v\left(r_{n}\right)}{v\left(r_{n-1}\right)} \leq e^{-(n-1)} .
$$

By Corollary $3.4, C_{\varphi}$ is compact.

On the other hand, for $n$ even

$$
\frac{w\left(r_{n}\right)}{w\left(\varphi\left(r_{n}\right)\right)}=\frac{w\left(r_{n}\right)}{w\left(r_{n-1}\right)}=\frac{v\left(r_{n}\right)}{v\left(p_{n}\right)} \geq e^{-1} .
$$

This completes the proof.

Theorem 3.3 yields a direct method to deduce the compactness of $C_{\varphi}$ in some weighted spaces once we know the compactness with respect to other weights. Precisely, assume that $v$ is essential and that $C_{\varphi}: H_{v}^{\infty} \rightarrow H_{w}^{\infty}$ is compact. If the weights $v$ and $\omega$ on $\mathbb{D}$ satisfy

$$
\frac{\omega}{\nu \circ \varphi} \leq C \frac{w}{v \circ \varphi}
$$

for some constant $C>0$, then $C_{\varphi}: H_{v}^{\infty} \rightarrow H_{\omega}^{\infty}$ is compact. Namely, the assumptions imply that (ii) of Theorem 3.3 is satisfied for $v$ and $w$, hence, by (9), it is satisfied by $v$ and $\omega$ as well. We especially have the following corollary.

COROLlaRY 3.6. Assume that $v$ and $w$ are typical weights, $v$ is essential, $v / w$ is increasing as $|z| \rightarrow 1, \varphi(0)=0$ and that $C_{\varphi}: H_{v}^{\infty} \rightarrow H_{v}^{\infty}$ is compact. Then also $C_{\varphi}: H_{w}^{\infty} \rightarrow H_{w}^{\infty}$ is compact.

PROOF. In this case (9) is equivalent to

$$
\frac{w}{w \circ \varphi} \leq C \frac{v}{v \circ \varphi}, \quad \text { or to } \quad \frac{v}{w} \circ \varphi \leq C \frac{v}{w} .
$$

But this holds with $C=1$ by the Schwarz lemma, since $v(z) / w(z)$ is radial and increasing as $|z| \rightarrow 1$.

THEOREM 3.7. Let $\varphi: \mathbb{D} \rightarrow \mathbb{D}$ be an analytic map such that $\partial \varphi(\mathbb{D}) \cap \partial \mathbb{D} \neq \emptyset$. Then there exists an essential typical weight $v$ such that $C_{\varphi}: H_{v}^{\infty} \rightarrow H_{v}^{\infty}$ is bounded but not compact.

COROllary 3.8. Let $\varphi: \mathbb{D} \rightarrow \mathbb{D}$ be an analytic map. The following assertions are equivalent:

(i) $C_{\varphi}: H_{v}^{\infty} \rightarrow H_{v}^{\infty}$ is compact for any (typical) weight $v$; 
(ii) $C_{\varphi}: H_{v}^{0} \rightarrow H_{v}^{0}$ is compact for any weight $v$ tending to zero at the boundary;

(iii) $C_{\varphi}: H^{\infty} \rightarrow H^{\infty}$ is compact;

(iv) $\varphi(\mathbb{D}) \subseteq r \mathbb{D}$ for some $r<1$.

ProOF OF THEOREM 3.7. The weight $w(z)=(1-|z|)$ is typical and essential and $C_{\varphi}: H_{w}^{\infty} \rightarrow H_{w}^{\infty}$ is always bounded by Theorem 2.3. Let us assume that $C_{\varphi}$ is compact in that case.

By Theorem 3.3, there is $r_{0}<1$ such that for any $r>r_{10}$ :

$$
1-|z|<\frac{1}{2}(1-|\varphi(z)|) \text { for }|z|=r .
$$

Let us define $M(r):=\sup _{|z|=r}|\varphi(z)|$, then we have

$$
1-r<\frac{1}{2}(1-M(r)) \text { for } r>r_{0} .
$$

The function $M(r)$ is non-decreasing, tends to 1 as $r \rightarrow 1^{-}$and, by the Hadamard Three Circle Theorem, it is logarithmically convex. In particular, $\log M(r) / \log r$ is non-decreasing as $r \rightarrow 1^{-}$. By (10), there is $\delta>1$ such that for $r \geq r_{0}$ we have $\log M(r) / \log r>\delta$. We define inductively a sequence $\left(r_{n}\right)_{n \in \mathbb{N}}$ such that $r_{n-1}=M\left(r_{n}\right)$. Obviously,

$$
r_{n-1}<r_{n}^{\delta} \text { for } n \in \mathbb{N}
$$

and $\lim _{n \rightarrow \infty} r_{n}=1$. We define an increasing function $u:[0,1) \rightarrow \mathbb{R}_{+}$which is equal to 1 on $\left[0, r_{0}\right], u\left(r_{n}\right)=2^{n}$ and it is affine on each interval $\left[r_{n-1}, r_{n}\right]$. The weight we are looking for is defined as $v(z)=1 / u(|z|)$.

Firstly, we show that $v$ is essential. By [BBT, Proposition 3.4], it suffices to prove the so-called condition (U) for $v$, that is, to find $\alpha>0$ and $C>0$ such that

$$
u(y)(1-y)^{\alpha} \leq C u(x)(1-x)^{\alpha} \text { for all } 0 \leq x<y<1 .
$$

We take $C=4$ and arbitrary $\alpha$ such that $\left(\left(1-r_{0}\right) /\left(1-r_{0}^{1 / \delta}\right)\right)^{\alpha}>2$. Indeed, for any $x, y$ either $u(y) \leq 2 u(x)$ or we find $n \leq k$ such that

$$
r_{n} \leq y, u(y) \leq 2 u\left(r_{n}\right) \text { and } x \leq r_{k}, u\left(r_{k}\right) \leq 2 u(x) .
$$

Thus

$$
\frac{u(y)}{u(x)} \leq 4 \frac{u\left(r_{n}\right)}{u\left(r_{k}\right)}<4\left(\frac{1-r_{0}}{1-r_{0}^{1 / \delta}}\right)^{\alpha(n-k)} .
$$

Since $g(t):=(1-t) /\left(1-t^{1 / \delta}\right)$ is an increasing function,

$$
\frac{1-r_{0}}{1-r_{0}^{1 / \delta}} \leq \frac{1-r_{i}}{1-r_{i}^{1 / \delta}} \quad \text { for any } \quad i \geq 0
$$


and, by (11), we get

$$
\frac{1-r_{0}}{1-r_{0}^{1 / \delta}} \leq \frac{1-r_{i}}{1-r_{i+1}}
$$

Combining with (13), we get

$$
\frac{u(y)}{u(x)} \leq 4 \prod_{i=k}^{n-1}\left(\frac{1-r_{i}}{1-r_{i+1}}\right)^{\alpha}=4\left(\frac{1-r_{k}}{1-r_{n}}\right)^{\alpha} \leq 4\left(\frac{1-x}{1-y}\right)^{\alpha},
$$

which gives (12) and $v$ is essential.

Now, for $|z|>r_{0}$ we find $n \in \mathbb{N}$ such that $r_{n} \leq|z|<r_{n+1}$, then

$$
\frac{v(z)}{v(\varphi(z))} \leq \frac{u(M(|z|))}{u(|z|)} \leq \frac{u\left(M\left(r_{n+1}\right)\right)}{u\left(r_{n}\right)}=1,
$$

by the definition of $\left(r_{n}\right)$. Thus $C_{\varphi}: H_{v}^{\infty} \rightarrow H_{v}^{\infty}$ is bounded by Proposition 2.1.

On the other hand, for any $n \in \mathbb{N}$ there is $z_{n},\left|z_{n}\right|=r_{n}$ such that $\left|\varphi\left(z_{n}\right)\right|=r_{n-1}$. Clearly,

$$
\frac{v\left(z_{n}\right)}{v\left(\varphi\left(z_{n}\right)\right)}=\frac{u\left(r_{n-1}\right)}{u\left(r_{n}\right)}=\frac{1}{2}
$$

and (since $\left|z_{n}\right| \rightarrow 1$ as $\left.n \rightarrow \infty\right) C_{\varphi}: H_{v}^{\infty} \rightarrow H_{v}^{\infty}$ is not compact by Corollary 3.4.

\section{Integral operators $C_{\varphi}$}

Unfortunately we are not able to give a characterization of nuclear, integral or absolutely summing composition operators for general weights. It is, however, not too complicated to find sufficient conditions for example as follows.

PROPOSITION 4.1. Let $1 \leq p<\infty, 1 / p+1 / q=1$, let $v$ and $w$ be radial weights and let $\varphi: \mathbb{D} \rightarrow \mathbb{D}$ be analytic. The operator $C_{\varphi}: H_{v}^{\infty} \rightarrow H_{w}^{\infty}$ is p-integral, if

$$
\sup _{z \in \mathbb{\mathbb { D }}} \int_{\xi \in \mathbb{D}} \frac{w(z)^{q}}{v \circ \varphi(\xi)^{q}|1-z \bar{\xi}|^{2 q}\left|\varphi^{\prime}(\xi)\right|^{2 q / p}} d A(\xi)<\infty .
$$

In the case $p=1$ the integral is replaced by a supremum in the usual way.

PROOF. Since the identity operator $H_{v}^{\infty} \rightarrow A_{v}^{p} \subset L^{p}(v(z) d A(z))$ is order bounded, it is enough to show that $C_{\varphi}: H_{v}^{\infty} \rightarrow H_{w}^{\infty}$ factorizes through the above defined map; see [DJT, Propositions 5.18 and 5.5]. To this end it suffices to prove that 
$C_{\varphi}: A_{v}^{p} \rightarrow H_{w}^{\infty}$ is bounded. Indeed, using the Bergman reproducing kernel ([Z, Section 4.1]) and the dominated convergence theorem,

$$
\begin{aligned}
\sup _{z \in \mathbb{D}}|f \circ \varphi(z)| w(z)=\sup _{z \in \mathbb{D}}\left|\int_{\mathbb{D}} \frac{f \circ \varphi(\xi)}{(1-z \bar{\xi})^{2}} d A(\xi)\right| w(z) \\
\leq \sup _{z \in \mathbb{D}}\left(\int_{\xi \in \mathbb{D}} \frac{w(z)^{q}}{\left.v \circ \varphi(\xi)^{q}|1-z \bar{\xi}|^{2 q}\left|J_{\varphi}(\xi)\right|^{-q / p} d A(\xi)\right)^{1 / q}}\right. \\
\quad \times\left(\int_{\mathbb{D}}|f \circ \varphi(\xi)|^{p} v \circ \varphi(\xi)^{p}\left|J_{\varphi}(\xi)\right| d A(\xi)\right)^{1 / p}
\end{aligned}
$$

where $\left|J_{\varphi}\right|$ is the 2-dimensional Jacobian determinant of $\varphi$. The identity $\left|J_{\varphi}\right|=\left|\varphi^{\prime}\right|^{2}$ and (14) permit us to conclude.

EXAMPLE 4.2. An example of integral composition operators.

Construction. Let $v(z):=(1-|z|)^{\alpha}$ and $w(z):=(1-|z|)^{\beta}$, where $\alpha, \beta>0$. Let $\Omega \subset \mathbb{D}$ be an open subset such that $\partial \Omega \cap \partial \mathbb{D}=\{1\}$. We also assume that $\partial \Omega$ is Dini-smooth ([Po, Theorem 3.5]) except at the point 1 where it has a Dini-smooth corner of opening $\gamma \pi$, where $\gamma<1$, in the sense of [Po, p. 51]; we make the technical assumption that for some $c>0$ the inequality

$$
|\operatorname{Im}(z)| \leq c(1-\operatorname{Re}(z))
$$

is satisfied for every $z \in \Omega$. Let $\varphi$ be a Riemann conformal mapping $\mathbb{D} \rightarrow \Omega$ such that $\lim _{z \rightarrow 1} \varphi(z)=1, \varphi(0)=0$ ([Po, Theorem 2.6]); for example $\varphi(z):=1-a(1-z)^{\gamma}$ for a suitable $a \in \mathbb{C}$. if

We show that (14) is satisfied for $p=1$ and the operator $C_{\varphi}$ is thus 1-integral,

(a) $\beta \geq 2 \geq(\alpha+2) \gamma$, or

(b) $\beta \geq(\alpha+2) \gamma \geq 2$.

By [Po, Theorem 3.9], there exists a neighbourhood $U$ of 1 such that for some constants $c, C>0$,

$$
\begin{aligned}
& c<\left|\frac{1-\varphi(z)}{(1-z)^{\gamma}}\right|<C \quad \text { for } z \in U, \text { and } \\
& c<\left|\frac{\varphi^{\prime}(z)}{(1-z)^{\gamma-1}}\right|<C \quad \text { for } z \in U,
\end{aligned}
$$


and the same estimates also hold for numbers $z$ outside $U$, because of $\overline{\varphi(\mathbb{D} \backslash U)} \subset \mathbb{D}$ and the Dini-smoothness assumption on $\partial \Omega$. From (17) we deduce that $\left|J_{\varphi}\right|=\left|\varphi^{\prime}\right|^{2}$ is bounded from below and above by a positive constant times $|1-z|^{2(\gamma-1)}$. Moreover, (15) implies that there exists a $C^{\prime}>0$ such that $|1-\xi| \leq C^{\prime}(1-|\xi|)$ for $\xi \in \Omega$; this and (16) imply that for $c^{\prime}>0,1-|\varphi(z)| \geq c^{\prime}|1-z|^{\gamma}$ for $z \in \mathbb{D}$. Hence, for $c^{\prime \prime}>0$

$$
v \circ \varphi(z)=(1-|\varphi(z)|)^{\alpha} \geq c^{\prime \prime}|1-z|^{\gamma \alpha} .
$$

Hence,

$$
\sup _{z . \xi \in \mathbb{D}} \frac{w(z)}{v \circ \varphi(\xi)|1-z \bar{\xi}|^{2}\left|\varphi^{\prime}(\xi)\right|} \leq C \sup _{z . \xi \in \mathbb{D}} \frac{(1-|z|)^{\beta}}{|1-\xi|^{(\alpha+2) \gamma-2}|1-z \bar{\xi}|^{2}} .
$$

We now distinguish between the two cases (a) and (b) mentioned above. If (a) holds, (19) is not larger than

$$
C \sup _{z, \xi \in \mathbb{D}} \frac{(1-|z|)^{\beta}}{|1-z \bar{\xi}|^{2}} \leq C \sup _{z \in \mathbb{D}} \frac{(1-|z|)^{\beta}}{(1-|z|)^{2}}<\infty .
$$

In the case (b) (19) can be estimated by

$$
C \sup _{\therefore . \xi \in \mathbb{D}} \frac{(1-|z|)^{\beta}}{(1-|\xi|)^{(\alpha+2) \gamma-2}|1-z \bar{\xi}|^{2}} .
$$

Clearly we can here take the sup only over the values $0<z, \xi<1$. The partial derivative of

$$
\frac{(1-z)^{\beta}}{(1-\xi)^{(\alpha+2) y-2}(1-z \xi)^{2}}, \quad 0<z, \xi<1,
$$

with respect to $\xi$ vanishes at the point $\xi=(((\alpha+2) \gamma-2+2 z) /((\alpha+2) \gamma z))^{\beta}$, and at this point $(20)$ becomes equal to a constant times $(1-z)^{\beta-(\alpha+2) \gamma} z^{(\alpha+2) \gamma-2}$. This expression is also finite, if the condition (b) is satisfied.

\section{References}

[BBT] K. D. Bierstedt, J. Bonet and J. Taskinen, 'Spaces of holomorphic functions with growth conditions and associated weights', Studia Math. (to appear).

[BS] K. D. Bierstedt and W. H. Summers, 'Biduals of weighted Banach spaces of analytic functions', J. Austral. Math. Soc. (Series A) 54 (1993), 70-79.

[C] J. Conway, Functions of one complex variable (Springer, Berlin, 1978).

[CM] C. Cowen, B. MacCluer, Composition operators on spaces of analytic functions (CRC Press, Boca Raton, 1995). 
[DJT] J. Diestel, H. Jarchow and A. Tonge, Absolutely summing operators, Cambridge Studies in Advanced Mathematics 43 (Cambrige University Press, Cambridge, 1995).

[J] H. Jarchow, 'Some functional analytic properties of composition operators', Quaestiones Math. 18 (1995), 229-256.

[L1] W. Lusky, 'On the structure of $H v_{0}(D)$ and $h v_{0}(D)$ ', Math. Nachr. 159 (1992), 279-289.

[L2] _ ' 'On weighted spaces of harmonic and holomorphic functions', J. London Math. Soc. 51 (1995), 309-320.

[Po] Ch. Pommerenke, Boundary behaviour of conformal maps, Grundlehren Math. Wiss. 299 (Springer, Berlin, 1992).

[RS] L. A. Rubel, A. L. Shields, 'The second duals of certain spaces of analytic functions', J. Austral. Math. Soc. 11 (1970), 276-280.

[Sh] J. H. Shapiro, Composition operators and classical function theory (Springer, Berlin, 1993).

[SWI] A. L. Shields, D. L. Williams, 'Bounded projections, duality, and multipliers in spaces of harmonic functions', J. Reine Angew. Math. 299/300 (1978), 256-279.

[SW2] - 'Bounded projections and the growth of harmonic conjugates in the disk', Michigan Math. J. 29 (1982), 3-25.

[SS] R. K. Singh and W. H. Summers, 'Composition operators on weighted spaces of continuous functions', J. Austral. Math. Soc. (Series A) 45 (1988), 303-319.

[T] J. Taskinen, Compact composition operators on general weighted spaces, Department of Mathematics, Univ. Helsinki Preprint 121, 1996.

[Z] K. Zhu, Operator theory in function spaces (Dekker, New York, 1995).

Dept. Matemática Aplicada

Univ. Politécnica de Valencia

E-46071 Valencia

Spain

e-mail: jbonet@pleiades.upv.es
Faculty of Matematics and Comp. Sci. A. Mickiewicz University, ul. Matejki $48 / 49$ 60-769 Poznań Poland e-mail: domanski@math.amu.edu.pl
Department of Mathematics Åbo Akademi University, FIN-20500

Åbo

Finland

e-mail: mlindstr@ra.abo.fi
Department of Mathematics

P. O. Box 4, FIN-00014

University of Helsinki

Finland

e-mail: taskinen@cc.helsinki.fi 